\title{
Cervical Cancer Prevention Efforts in India
}

\author{
Neerja Bhatla ${ }^{1} \cdot$ Jyoti Meena ${ }^{1} \cdot$ Sarita Kumari ${ }^{1} \cdot$ Dipanwita Banerjee $^{1} \cdot$ Prerana Singh $^{2} \cdot$ Jayashree Natarajan $^{3}$
}

Published online: 2 June 2021

(C) Association of Gynecologic Oncologists of India 2021

\begin{abstract}
Purpose Cervical cancer is one of the leading cancers among women in India. Its prevention and control require a concerted effort to improve awareness among women regarding primary and secondary prevention strategies as well as access to care for treatment and palliation. A focused strategy is important to reach the World Health Organization's targets for cervical cancer elimination, due to be completed by 2030 .

Methods Currently available literature was reviewed regarding cervical cancer prevention strategies in India including various national programmes and other initiatives on the part of government, non-governmental organizations and professional organizations. Their applicability to the present situation was assessed.

Results National programmes need to build on success stories of various states and neighbouring countries as well as to audit the performance. Strengthening of cancer registries and improvement of linkages between different healthcare levels with incorporation of task-shifting, adding digital technology and supporting programmes that promote women's welfare and health will also provide synergy to cancer control programmes. In the current pandemic era, HPV self-sampling can be an ideal method for screening. The development of an affordable, point-of-care HPV test is urgently needed to facilitate its introduction in low- and middle-income countries. HPV vaccination efforts need to be speeded up.

Conclusion Scaling up of cervical cancer prevention with inclusion of widespread HPV vaccination and primary HPV test should be the new standard of care.
\end{abstract}

Keywords Cervical cancer $\cdot$ Prevention $\cdot$ Vaccination $\cdot$ Screening $\cdot$ HPV $\cdot$ CIN

Neerja Bhatla

neerja.bhatla07@gmail.com

Jyoti Meena

drjyotirajesh@gmail.com

Sarita Kumari

sarita2325@gmail.com

Dipanwita Banerjee

dr.dipanwita@yahoo.co.in

Prerana Singh

dr.amitanshu@gmail.com

Jayashree Natarajan

vasanthjayashree@gmail.com

1 Department of Obstetrics and Gynaecology, All India Institute of Medical Sciences, New Delhi 110029, India

2 Department of Obstetrics and Gynaecology, Maa Janki Hospital and Research Center, Muzaffarpur, Bihar 842002, India

3 Department of Gynaecologic Oncology, Cancer Institute (WIA) Adyar, Chennai, Tamil Nadu 600020, India

\section{Introduction}

Cervical cancer is the second most common cancer among women in India, which contributes to one-fifth of the global burden. In 2020, Globocan estimated 123,907 incident cases and 77,348 deaths, with an age-standardized incidence rate of 18 per 100,000 women and a cumulative risk of $2.01 \%$ [1]. The 5-year relative survival rate of approximately $46 \%$ (range 34-60\%) is much lower than that of other Asian countries [2]. This is due to the fact that cervical cancer is diagnosed at advanced stage in more than $80 \%$ cases, resulting in high mortality [3].

Cervical cancer prevention efforts by both the government and voluntary organizations have been ongoing since several decades but are yet to make an impact. While only screening and treatment of precancerous lesions was possible initially, presently there is an additional option of human papillomavirus (HPV) vaccination. There are approximately 59.7 million girls and 272.8 million women in India in the eligible age group for cervical cancer 
vaccination and screening, respectively [4]. In this article, we review the progress of cervical cancer prevention efforts in India, including the indirect impact of social reforms on factors affecting the burden of this preventable disease.

\section{Epidemiology of Cervical Cancer}

The natural history of cervical cancer is well documented. Persistent infection with high-risk (oncogenic) types of human papillomavirus (hrHPV) is the necessary cause of cervical cancer, with several co-factors that facilitate initiation and progression, including early age at coitarche, multiple sexual partners (self or spouse), multiple pregnancies, associated sexually transmitted infections, etc. HPV belongs to a family of small double-stranded DNA viruses, and more than 100 distinct types have been identified. HPV 16 and 18 account for over $70 \%$ of invasive cervical cancers globally. In India, over $80 \%$ of cervical cancer and $63 \%$ of high-grade lesions are linked with infection due to HPV 16 and 18, which is greater than the global average [5]. These are the two types contained in all prophylactic HPV vaccines.

Cervical cancer has a long pre-invasive phase that lasts for 10-15 years. This provides a window of opportunity to detect and treat the neoplasia in pre-invasive stages by simple outpatient treatment modalities, as well as early detection of cancers. The incidence and mortality of this disease are a reflection of the available resources and medical infrastructure for population-wide screening and treatment.

\section{WHO Call for Elimination of Cervical Cancer}

In May 2018, the World Health Organization (WHO) issued a call for elimination of cervical cancer as a public health problem through widespread HPV vaccination, screening, early diagnosis and treatment of cervical precancer and cancer. On November 17, 2020, the World Health Assembly (WHA) formally launched a global strategy for its implementation. Even in the midst of the COVID-19 pandemic, 194 countries around the world confirmed their support for this important task [6]. The elimination programme aims to achieve the following targets by 2030:

- $90 \%$ girls fully vaccinated by 15 years of age with two doses of HPV vaccine;

- $70 \%$ women screened with a high-performance test at 35 and 45 years of age; and,
- $90 \%$ of women with cervical pre-cancer and cancer receive treatment to achieve a goal of less than four cases per 100,000 women.

The United Nations' Sustainable Development Goals for 2030 aim to reduce the premature mortality from noncommunicable diseases by one-third through prevention and treatment. Achieving the elimination targets will help to achieve this goal as well.

\section{National Programmes for Cancer Control}

Implementation of both arms of cervical cancer prevention, i.e., HPV vaccination and screening linked with treatment of pre-cancers, can lead to significant reduction in incidence and mortality to a level where it will no longer be a public health problem. Factors like cultural barriers, lack of awareness, low socio-economic status, poor access to healthcare, and lack of resources and infrastructure also need to be addressed.

The National Cancer Control Programme (NCCP) was first started in 1976. The primary objective was prevention of cancers through health education; and secondarily prevention of cervical oral and breast cancers by screening, strengthening the existing cancer treatment facilities and providing palliative care to patients at terminal stage. In 2010, NCCP was integrated with the National Programme for Prevention and Control of Diabetes, Cardiovascular Diseases and Stroke (NPDCS). The programme was implemented across 21 states in 100 districts during the year 2010-2012. The review of initial phase of programme helped in identifying the bottlenecks and accordingly the programme was re-strategized and scaled-up. For activities up to district level, states are given financial support under the umbrella of the national health mission (NHM). Noncommunicable diseases (NCD) clinics were established at District and Community Health Centers (CHC) for early diagnosis, treatment and follow-up services for common NCDs. For patients attending these NCD clinics, provision to provide free diagnostic facilities and drugs has been made under the programme. Beyond the district level, there is Tertiary Care Cancer Centers (TCCCs) scheme; aim of this scheme is to strengthen/set up State Cancer Institutes (SCI) and TCCCs to provide comprehensive cancer care. The programme strategy implemented at various levels is as follows:

\section{(i) Primary level}

- Door to door information, education and communication (IEC) by Accredited Social Health Activists (ASHA) and provision of IEC material 
- Monthly visits by medical officer (MO) to subcentre to monitor ASHA's work and record keeping

- Periodic training of health workers in screening

- Mass recruiting campaign and periodical screening camps by $\mathrm{MO} /$ health staff of each subcentre

- Utilization of laboratory technician and health workers for screening with cytology/HPV at primary health centres (PHCs)

\section{(ii) Secondary level}

- Provision of

- A gynaecologist trained in colposcopy at community health centre (CHC) level

- Colposcopy equipment (1-3 per district) and a thermal/cryoablation unit

- A pathologist

- Chemotherapy and palliative care services

- District level periodic monitoring and data keeping

\section{(iii) Tertiary level}

- Improving training in Regional Cancer Centers (RCC) and training institutes (emphasis on surgical skills)

- Infrastructure for radiation and imaging techniques.

\section{Cancer Registries}

The National Cancer Registry Programme (NRCP) was initiated in 1982 by the Indian Council of Medical Research (ICMR), and it provides a picture of the magnitude and patterns of cancer. The cancer registries are either population-based or hospital-based and provide data to ICMR on a regular basis. The first rural cancer registry was initiated at Barshi, Maharashtra, in 1987. This activity not only increased the cancer awareness among the villagers but also the frequency of early detection and significantly decreased the deaths due to cervical cancer [7]. It was further extended to other districts of Maharashtra and Gujarat. At present there are 36 population-based and 236 hospital-based cancer registries under NRCP.

\section{Cervical Cancer Screening Efforts in India}

To reduce the incidence and mortality due to cervical cancer, even a single round of screening is effective compared to no screening [8]. The incidence is higher in the rural population where the majority of women are socio-economically disadvantaged, with no formal education and no awareness of the risk factors associated with development of the disease. Knowledge, attitude and practice (KAP) surveys conducted among rural women have shown better awareness and knowledge among younger and literate women compared to older and illiterate ones [9].

Different screening strategies have been used to reduce the incidence of disease especially among rural population.

\section{Screening Camps}

This approach of organizing outreach clinics was followed over decades where women voluntarily approach the camp for screening when provided with awareness regarding cervical cancer. It was useful for women who actively participated in the screening programmes. It did not, however, translate into active community participation. The apprehension to undergo internal examination during screening test leads to poor acceptance and participation. Initial cytology-based screening approach also leads to multiple dropouts at various steps. A study by Nene et al., in the rural population of Barshi Tehsil of Maharashtra, between 1982 and 1987 showed that mere conductance of camps was not sufficient to motivate women for screening [10]. However, another study conducted by Sharma et al., among Delhi population, showed encouraging results for a camp-based approach and emphasized the importance of regular cervical cancer screening through this approach all over the country [11].

\section{Screening Through Village Health Nurses (VHNs)}

The Tamil Nadu Health Services Project for cervical cancer screening trained VHNs in screening for non-communicable diseases (NCDs) in the screening programme. The project showed that using trained health workers for screening is feasible and effective [12]. Lessons learnt from this programme formed the basis for the NPCDCS strategy.

\section{Cervical Cancer Screening Modalities}

Several screening methods, such as cytology, co-testing (HPV + cytology), primary HPV testing, and visual inspection with acetic acid, are being utilized in various settings depending on the availability of resources and compliance. The Federation of Obstetricians and Gynaecologists of India (FOGSI) has published good clinical practice recommendations (GCPR) for screening and management of screen-positive women in different resource settings. 


\section{Cytology (Pap Smear)}

It is the most common modality used so far in India in urban areas. It has been found to reduce the incidence of cervical cancer by about $80 \%$ in developed countries [13]. However, it requires considerable infrastructure and funds along with repeated rounds of testing due to poor sensitivity.

\section{Visual Inspection with Acetic Acid (VIA)}

VIA has a sensitivity comparable to cytology and has been implemented in rural areas taking into consideration the lack of facilities for cytology. Studies on efficacy of VIA have shown that it reduces the mortality due to cervical cancer [14]. A cluster randomized study conducted at Dindigul district in Tamil Nadu, India, showed reduction in incidence by $25 \%$ and mortality by $35 \%$ over a follow-up period of 7 years [15]. The NPCDCS guidelines published in 2016 have adopted VIA as the test of choice in screening programmes, with 5-yearly screening among women aged 30-65 years, along with screening for breast and oral cancer. FOGSI also recommends VIA as the test of choice in limited resource settings [16].

\section{HPV Testing}

HPV testing has been accepted as the best screening test with highest sensitivity and negative predictive value. A population-based study in a peri-urban community in Andhra Pradesh by Gravitt et al. found the sensitivity and specificity of HPV test to be higher compared to Pap and VIA. [17]. For one or two rounds of screening, an affordable point-of-care HPV test remains the ideal option in developing countries. CBNAAT-based testing systems developed during the COVID pandemic may prove useful for HPV testing too.

Studies have shown that HPV self-sampling results are comparable to provider-collected sample in detection of cervical neoplasia [18, 19]. Newer innovations like batteryoperated portable colposcopes; battery-operated thermal ablators and artificial intelligence-based software applications to detect and triage the pre-cancerous lesions are rapidly progressing and will be helpful to implement screening on a large scale.

Until HPV tests are available, the momentum gained using VIA should not be lost. In limited resource countries like Bangladesh and India where disease burden is high, VIA-based screening programmes were introduced with varying degrees of programmatic organization and performance. The affordability and simplicity of VIA test helped in training a large number of healthcare personnel for screening and led to development of a multilevel coordination within the healthcare system [20].

For a successful screening programme, quality assurance of services is also important. In studies from Bangladesh and India, it has been observed that following the right approach to organize several components and meticulous attention to quality is crucial for the success of a screening programme and not merely the choice of a good screening test [20]. The selected screening strategy must be feasible, simple, safe, accurate, acceptable, and easily accessible to the highest-risk women. However, screening alone is not sufficient if follow-up is poor for screen positive women. Vidhubala et al. conducted a study to evaluate community-based screening programme in South India in a rural setting (Tirunelveli and Tuticorin districts). The data from case records of 2192 women who underwent conventional Pap smear and VIA were evaluated. They found that among 807 women who were referred only $9.2 \%$ (74) visited the referral centre. They concluded that the success of screening programme depends on the completion of care continuum, and fragmentation to this accounts for poor follow-up after screening [21].

\section{HPV Vaccination}

According to the WHO position paper on HPV vaccination, vaccinating HPV-naive girls is cost-effective and is particularly relevant in resource constrained settings [22]. HPV vaccines have been licensed in India since 2008 for females aged 9 to 45 years and the National Technical Advisory Group on Immunization (NTAGI) has approved their use. The Indian Academy of Paediatrics Committee on Immunisation (IAPCOI) recommends it for all females starting from 9 years of age. FOGSI recommends 9-14 years as the ideal time for vaccination, with vaccination of older age cohorts to be considered with an understanding that vaccination in sexually active females may be less effective as they may already be infected, but may provide some benefit against the types to which they have not been exposed earlier [16].

A large multicentre cohort Indian study of the immunogenicity of two versus three doses of qHPV vaccine found it to be non-inferior. Even single-dose recipients showed a robust and sustained immune response however, it was inferior to that after two or three doses and the antibody levels were stable over a period of 4 years [23]. Currently trials are undergoing to address the possibility of single dose vaccine. 


\section{Implementation of HPV Vaccination Programme in India}

Delhi was the first state in India to implement opportunistic HPV vaccination for school girls aged 11-13 years in 2016 [24]. No severe adverse events were observed, but the programme was very limited in its reach. Subsequent to this the Government of Punjab developed operational guidelines for implementing HPV vaccination through health facilities with technical inputs of ICMR, WHO, and UNICEF. They adopted a campaign mode in two districts with the highest burden: Bathinda (incidence 17.5 per $100,000)$ and Mansa $(17.3$ per 100,000). Phase 1 started in November 2016, vaccination coverage was excellent and 98\% (9672/9922) of the target population completed two doses in government and government-aided schools. Upon completion of phase 2 in November 2017, first dose was received by $94 \%(15,140 / 16,106)$ eligible girls and $99 \%$ $(14,988 / 15,140)$ received the second dose [25]. This paved the way for policy makers to consider HPV vaccination in other states.

Sikkim was the first state to completely vaccinate girls aged 9-14 years in the entire state in 2018. It targeted 25,284 girls in 1166 schools; first dose was received by $97 \%$ girls at school or in a health centre (for individuals not attending school) with a second dose 6 months later. Minor adverse events were noted such as headache, dizziness, nausea, pain at injection site, which were temporary and resolved quickly [25]. This was followed subsequently by inclusion of vaccination of girls at nine years in the routine immunization schedule. WHO, UNICEF, Jhpiego and state coordinated advisory committee provided technical support for the programme.

\section{Changing Demography and Risk Factors for Cervical Cancer}

Child marriage has been prohibited in India by legislation (The Child Marriage Restraint Act, 1929) and the legal age of marriage is 18 years. The current mean age at marriage is 21.2 years, with $2.6 \%$ women getting married before the legal age [4]. Promoting education of the girl-child, improvement of her nutritional status and encouraging women to get married only after securing education and occupation has brought considerable changes in the sociodemographic pattern. Women education and health are promoted through government policies (Kishori Shakti Yojna, Rajiv Gandhi scheme for empowerment of adolescent girls-SABLA). The number of literate women has increased steeply over the past 3 decades, and country-wide female literacy rate is $70.3 \%$, compared to $84.7 \%$ for males
[4]. Small family norms are motivated through incentives for family planning. The $\mathrm{RMNCH}+\mathrm{A}$ (Reproductive Maternal Neonatal Childhood Health + Adolescent) strategy of NHM includes contraceptive delivery at home by ASHA workers, delaying the age at marriage, promoting menstrual hygiene through the Menstrual Hygiene Scheme, sexual hygiene awareness and prevention and treatment of reproductive tract illnesses in "Suraksha clinics". Opportunities for women to pursue their professional and career related goals are promoted by encouraging women who are working to establish small-scale industries and selfemployment.

\section{Contribution of Professional Organizations and Non-governmental Organizations (NGOs)}

Professional organizations like FOGSI, Asia-Oceania Research Organization in Genital Infection and Neoplasia (AOGIN-India), Indian Society of Colposcopy and Cervical Pathology (ISCCP) and Association of Gynaecologic Oncologists of India (AGOI) have contributed to cervical cancer prevention strategies by developing screening guidelines, conducting screening outreach camps and conducting awareness talks across the country, using innovative strategies like the Lifeline Express to reach remote areas and collaborating with corporate and paramedical organizations.

\section{Future Prospects}

India's efforts for cervical cancer control should take advantage of public-private partnerships involving global organizations like $\mathrm{WHO}$ and professional organizations such as FOGSI, ISCCP, and AOGIN-India, in collaboration with corporates as part of their social responsibility commitments. WHO has recommended two rounds of HPV testing by the ages 35 and 45 years [6]. In the current pandemic era, where prevention efforts have had a setback, HPV self-sampling can be an ideal method, avoiding crowding of health facilities and minimizing contact with health personnel.

\section{Conclusion}

Introduction of non-cytological screening by HPV test and VIA has brought a paradigm shift in the cervical cancer screening. Over the last decade, HPV vaccination has multiplied the efforts. In spite of all advancements, screening for cervical cancer will still be required as 
millions of women have already been exposed to the virus. Inclusion of widespread vaccination and HPV test as a point-of-care test should be the new standard of care in cervical cancer prevention.

\section{Funding Nil.}

\section{Declarations}

Conflict of interest The authors declare that they have no conflict of interest.

\section{References}

1. Sung H, Ferlay J, Seigel RL, Laversanne M, Soerjomataram I, Jemal A, et al. Global cancer statistics 2020: GLOBOCAN estimates of incidence and mortality worldwide for 36 cancers in 185 countries. CA Cancer J Clin. 2021;71:209-49.

2. Sankaranarayanan R, Swaminathan R, Lucas E. Cancer survival in Africa, Asia, Caribbean and Central America: database and attributes. IARC Sci Publ. 2011;162:23-31.

3. World Health Organization (WHO). Comprehensive cervical cancer control: a guide to essential practice. Geneva, Switzerland: World Health Organization; 2014.

4. Office of the Registrar General and Census Commissioner, India. Ministry of Home Affairs, Government of India. https://cen susindia.gov.in/. Accessed May 102021.

5. Bruni L, Albero G, Serrano B, Mena M, Gomez D, Munoz J, et al. Human Papillomavirus and related diseases report. ICO Information Centre on HPV and Cancer (HPV Information Centre); 2016. https://hpvcentre.net/statistics/reports/XWX.pdf. Accessed May 22021.

6. World Health Organization. Global strategy to accelerate the elimination of cervical cancer as a public health problem. Geneva. 2020. https://www.who.int/publications/i/item/ 9789240014107. Accessed April 172021.

7. Jayant K, Nene BM, Badwe RA, Panse NS, Thorat RV, Khan FY. Rural cancer registry at Barshi, Maharashtra and its impact on cancer control. Natl Med J India. 2010;23:274-7.

8. Srivastava AN, Misra JS, Srivastava S, Das BC, Gupta S. Cervical cancer screening in rural India: status and current concepts. Indian J Med Res. 2018;148:686-96.

9. Weinberg CR. HPV screening for cervical cancer in rural India. N Engl J Med. 2009;361:305-6.

10. Nene BM, Jayant K, Malvi SG, Dale PS, Deshpande R. Experience in screening for cervical cancer in rural areas of Barshi Tahsil (Maharashtra). Indian J Cancer. 1994;31:34-40.

11. Sharma P, Rahi M, Lal P. A community-based cervical cancer screening program among women of Delhi using camp approach. Indian J Community Med. 2010;35:86-8.

12. Gajalakshmi CK, Krishnamurthi S, Ananth R, Shanta V. Cervical cancer screening in Tamilnadu, India: a feasibility study of training the village health nurse. Cancer Causes Control. 1996;7:520-4.
13. Miller AB, Nazeer S, Fonn S, Brandup-Lukanow A, Rehman R, Cronje $\mathrm{H}$, et al. Report on consensus conference on cervical cancer screening and management. Int J Cancer. 2000;86:440-7.

14. Aggarwal P, Batra S, Gandhi G, Zutshi V. Comparison of Papanicolaou test with visual detection tests in screening for cervical cancer and developing the optimal strategy for low resource settings. Int J Gynecol Cancer. 2010;20:862-8.

15. Sankaranarayanan R, Esmy PO, Rajkumar R, Muwonge R, Swaminathan R, Shanthakumari $S$, et al. Effect of visual screening on cervical cancer incidence and mortality in Tamil Nadu, India: a cluster-randomised trial. Lancet. 2007;370:398-406.

16. Bhatla N, Singhal S, Saraiya U, Srivastava S, Bhalerao S, Shamsunder $S$, et al. Screening and management of preinvasive lesions of the cervix: Good clinical practice recommendations from the Federation of Obstetrics and Gynaecologic Societies of India (FOGSI). J Obstet Gynaecol Res. 2019;46:201-14.

17. Gravitt PE, Paul P, Katki HA, Vendantham H, Ramakrishna G, Sudula M, et al. Effectiveness of VIA, Pap, and HPV DNA testing in a cervical cancer screening program in a peri-urban community in Andhra Pradesh, India. PLoS ONE. 2010;5:e13711.

18. Petignat $\mathrm{P}$, Faltin DL, Bruchim I, Tramèr MR, Franco EL, Coutlée F. Are self-collected samples comparable to physiciancollected cervical specimens for human papillomavirus DNA testing? A systematic review and meta-analysis. Gynecol Oncol. 2007;105:530-5.

19. Bhatla N, Dar L, Patro AR, Kumar P, Kriplani A, Gulati A, et al. Can human papillomavirus DNA testing of self-collected vaginal samples compare with physician-collected cervical samples and cytology for cervical cancer screening in developing countries? Cancer Epidemiol. 2009;33:446-50.

20. Bhatla N, Nessa A, Oswal K, Vashist S, Sebastian P, Basu P. Program organization rather than choice of test determines success of cervical cancer screening: case studies from Bangladesh and India. Int J Gynecol Obstet. 2020;152:40-7.

21. Vidhubala E, Niraimathi K, Shewade HD, Mahadevan S. Cervical cancer care continuum in South India: Evidence from a community-based screening program. J Epidemiol Glob Health. 2020;10:28-35.

22. World Health Organization. Human papillomavirus vaccines: WHO position paper, May 2017. Wkly Epidemiol Rec No. 2017;19(92):241-68.

23. Sankaranarayanan R, Prabhu PR, Pawlita M, Gheit T, Bhatla N, Muwonge R, et al. Immunogenicity and HPV infection after one, two, and three doses of quadrivalent HPV vaccine in girls in India: a multicentre prospective cohort study. Lancet Oncol. 2016;17:67-77.

24. Mehrotra R, Hariprasad R, Rajaraman P, Mahajan V, Grover R, Kaur $P$, et al. Stemming the wave of cervical cancer: human papillomavirus vaccine introduction in India. $\mathbf{J}$ Glob Oncol. $2018 ; 4: 1-4$.

25. Sankaranarayanan R, Basu P, Kaur P, Rajesh B, Singh GB, Denzongpa $\mathrm{P}$, et al. Current status of human papillomavirus vaccination in India's cervical cancer prevention efforts. Lancet Oncol. 2019;20:e637-44.

Publisher's Note Springer Nature remains neutral with regard to jurisdictional claims in published maps and institutional affiliations. 